\title{
Histological, immunofluorescent, and ultrastructural features of lymphogranuloma venereum:
}

\author{
A case report
}

\author{
B ALACOQUE,* H CLOPPET,† C DUMONTEL, $\neq$ AND G MOULIN* \\ From the *Department of Dermatovenereology, Hopital de l'Antiquaille, Lyon, the †Department of \\ Immunochemistry, Institut Pasteur, Lyon, and the $\neq$ Department of Histology, A Carrel Medical \\ University, Lyon, France
}

SUMMARY We studied the course of a case of lymphogranuloma venereum (LGV) over two years. The comparative histological, immunological, and ultrastructural studies showed the existence of characteristic granulations within the macrophages of the granuloma.

We suggest that direct immunofluorescence is a specific method for diagnosing cutaneous LGV. Some ultrastructural aspects lead us to believe that different chlamydial bodies exist inside the granuloma.

\section{Introduction}

To diagnose lymphogranuloma venereum (LGV) a number of criteria are necessary: characteristic clinical symptoms, compatible histology, and positive results to serological tests for chlamydial (LGV serotypes) antibodies. Electron microscopy can be a useful adjunct to diagnosis, but is not routinely performed. The most specific diagnostic test is culture of the organism from material obtained from the patient. If culture is negative, diagnosis can only be presumptive. We have tried to provide a specific test based on direct immunofluorescence of tissue samples. Our ultrastructural study provided evidence of atypical chlamydial bodies during their life cycle in LGV.

\section{Patient and methods}

A 22 year old Italian women was admitted to hospital in May 1980 for investigation of condylomatous perineal lesions that had developed two years previously. There was no relevant history; she had not travelled outside Europe, and had not had extramarital sexual intercourse. The condition had started in April 1978 during the second month of her first pregnancy, when three or four painful, purulent, punctiform papules developed. The lesions were found on the

Address for reprints: Dr B Alacoque, Department of Dermatovenereology, Hopital de l'Antiquaille, 1 Rue de l'Antiquaille, 69321 Lyon cedex 1, France

Accepted for publication 12 March 1984 external and inferior aspects of the left labium majus, which was oedematous, erythematous, and painful but not indurated. There were no systemic symptoms. Because the lesions spread to the whole vulvar area her baby was delivered by cesarean section in October 1978. The course of the pregnancy had been normal, and the neonate was normal. After the birth the lesions became hypertrophic and condylomatous and spread to the perineo-anal region. There were no urinary or gastrointestinal symptoms, but she had dyspareunia. No diagnosis was made. Different medical treatments were ineffective. The patient consulted us in May 1980.

Examination showed vulvoperineal esthiomene (fig 1). The lesions were composed of large condylomatous formations $(2 \mathrm{~cm}$ in diameter), erythematous papules (1 $\mathrm{mm}$ in diameter) disseminated over healthy and condylomatous skin, and papular vesicles resulting from ectasia of the lymphatic vessels. On the vulva the condylomata were on the external aspect of the labia majora; the vaginal orifice and labia minora were unaffected but somewhat oedematous. Smaller identical lesions were found around the gluteal fold and the anal margin. General and proctological examination showed no abnormalities, and the vagina was normal. Treatment with sulphonamides, oxytetracycline, and erythromycin produced resolution of the dyspareunia, but little improvement in the condylomatous lesions.

\section{LABORATORY FINDINGS}

Results of complement fixation tests for LGV were $<1 / 8$, and of microimmunofluorescence tests $<1 / 40$. 
Urethral cultures failed to yield chlamydiae. Biopsy specimens were not cultured. Other bacteriological, parasitological, and virological cultures and serological tests gave negative results.

\section{INVESTIGATIONS}

Bipedal lymphography was normal. Kinmonth's method with blue patent violet showed a quick and erratic impregnation of the ectasia of the lymphatic vessels.

Several biopsies of the vulvar esthiomene were performed using the following stains and methods: haematoxylin, eosin, and safran; May-GrünwaldGiemsa, immunoperoxidase followed by MayGrünwald-Giemsa; direct immunofluorescence immunoperoxidase; and electron microscopy.

\section{Immunofluorescence}

The fluorescent antibody technique was performed on deparaffinised $5 \mu \mathrm{m}$ thick sections. ${ }^{1}$ Antiserum to Chlamydia trachomatis was obtained either from a patient with chlamydial urethritis (test dilution 1/10) or from hyperimmune rabbits (test dilution 1/100) kindly supplied by $\mathrm{Dr}$ Thouvenot, Faculty of Medecin, A Carrel Medical University, Lyon. Fluorescent antiserum was respectively goat antihuman IgG (Institut Pasteur, Paris, France) diluted 1/100 and antirabbit IgG (Institut Pasteur) diluted $1 / 100$. The specificity of the tests was controlled by a fluorescence inhibition procedure by previous incubation of some sections with heterologous antichlamydial reagent. The specificity was also controlled by incubation with immunofluorescent conjugate after omission of the human and rabbit immune serum respectively. All dilutions were performed in phosphate buffered saline (PBS) ph $\mathbf{7} \cdot \mathbf{2}$.

\section{Immunoperoxidase procedure}

The immunocytological reaction was performed on either paraffin sections or semithin sections. Serial sections of $5 \mu \mathrm{m}$ paraffin sections were mounted on glass slides with aqueous gelatin, deparaffinised in toluene, and rehydrated. Semithin $(1 \mu \mathrm{m})$ sections were obtained from the samples used for the ultrastructural study. They were mounted on gelatin precoated glass slides. To remove Araldite, the sections were exposed to ethanolic sodium hydroxide diluted $1 / 1$ with $100 \%$ ethanol for 10 minutes. After three washes in $100 \%$ ethanol, the sections were rehydrated and then bleached for 10 minutes in $0.03 \%$ hydrogen peroxide.

The sections were stained by an indirect immunoperoxidase method. They were incubated overnight with the patient's immune serum diluted $1 / 10$ to $1 / 80$ in trometamol (TRIS) buffered saline (ph 7.6), followed by incubation with sheep antihuman IgG conjugated with peroxidase (Institut Pasteur) diluted $1 / 100$. The peroxidase substrate was hydrogen peroxide 3-3' diamino-benzidine. The sections were mounted with Entellan (E Merck, Darmstad, West Germany). This study was controlled by the incubation of sections with sheep antihuman IgG labelled with peroxidase after omitting the human immune serum or substituting for it a non-immune human serum at the same dilution.

\section{Electron microscopy}

Pieces of condylomatous lesions were removed and cut into small pieces. They were fixed separately in 4\% glutaraldehyde and $2 \%$ formalin buffered with cacodylate $0 \cdot 32 \mathrm{~mol} / \mathrm{l}(\mathrm{ph} 7 \cdot 4)$. They were post-fixed in $2 \%$ osmium tetroxide buffered at ph $7 \cdot 4$. After being washed, tissues were dehydrated in graded ethanol and embedded in Araldite. Ultrathin sections prepared with a Reichert OMU 3 were contrasted with uranyl acetate and lead citrate. They were examined and photographed in a Jeol JEM 7 electron microscope.

\section{Results}

\section{HISTOLOGY}

In some areas sections stained with haematoxylin, eosin, and safran showed acanthosis of the epidermis with noticeable papillomatosis; in other areas the epidermis was almost normal. The papillary dermis was oedematous and had dilated lymphatic vessels. The reticular dermis presented a very dense fibrous appearance. It contained inflammatory cell infiltrates, which were often in clusters throughout the dermis but were most frequently located around the vessels. The inflammatory granuloma was polymorphous, with lymphocytes, plasma cells, and sometimes multinucleated giant cells. The infiltrates were found as far down as the deep dermis close to the sweat glands. Free pigment was present in the dermis.

Sections stained with May-Grünwald-Gienssa showed features generally similar to those described above. Many mast cells were seen disseminated inside the granuloma. Their cytoplasm was full of small, spherical, blue granulations. These granulations were sometimes disseminated in the tissue or formed clusters far from or next to the mast cells. Their acidophilic colour was more prominent outside the cell. In addition to the numerous mast cells, a few macrophages were seen. They were located near the dilated lymphatic vessels either alone or in clusters of two to 10 cells in the vicinity of the inflammatory cells. The cytoplasm of these macrophages was filled with large granulations. They were $0.4 \mu \mathrm{m}$ to $3.2 \mu \mathrm{m}$ in size, irregularly shaped, and in contrast to the 

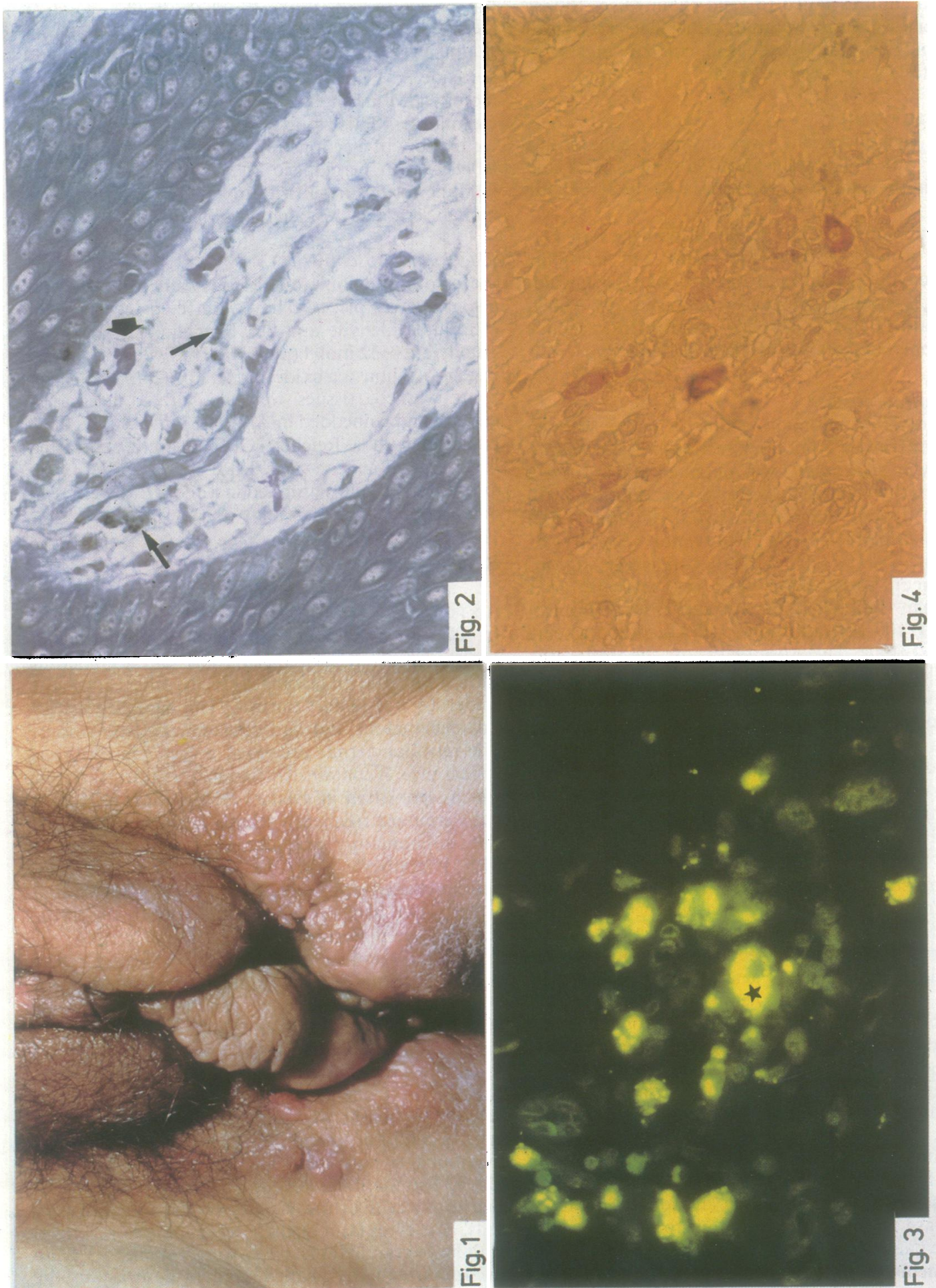

\section{느}

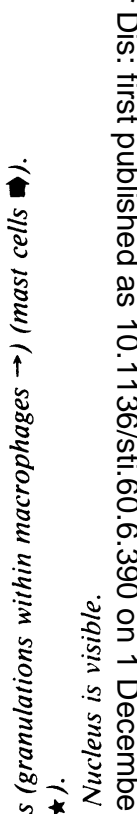

o

$\supseteqq \cong$ ऽั ฐ วิำ 乌 ○े 응 ติ

$\times$ ํํㅇㅇ 오옹 농 음

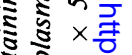
क $8 \frac{1}{4}$ 동ำ

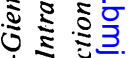
1

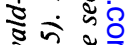

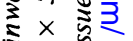
ป 8 : 过 इ ¿

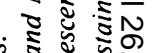

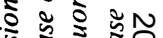
\& 8 . वे ำ วิ รับ ำ ำ ई. ई वे ๘

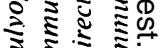
今 m $\mathrm{Nm}$ 西 б L I L 


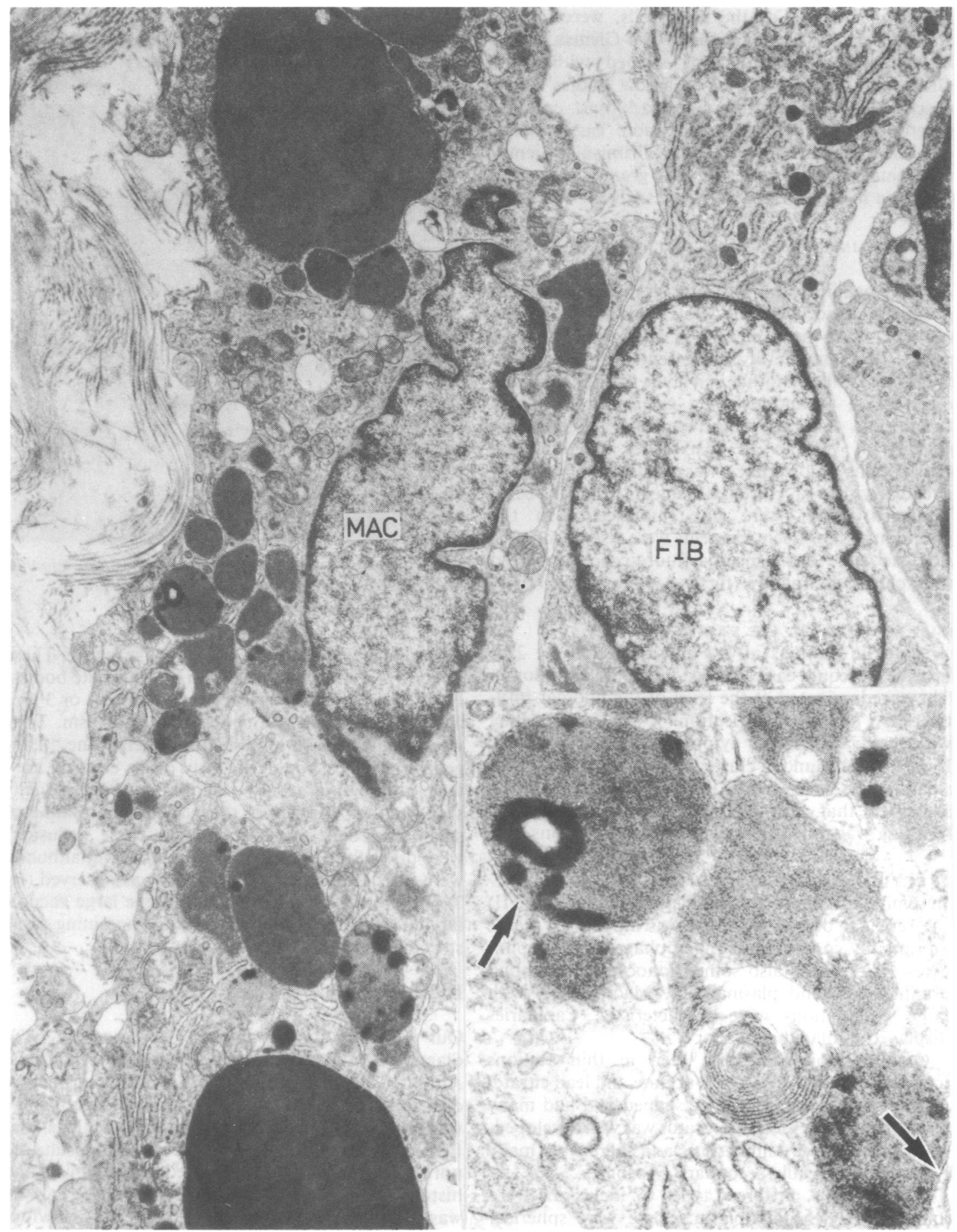

FIG 5 Macrophage with large atypical inclusions $(\times 12600)(F I B=$ fibroblast, $M A C=$ macrophage. Insert: Double unit membrane limiting the inclusions $\rightarrow(\times 65000)$. 
azurophil staining of the mast cells, were heavily stained dark blue by May-Grünwald-Giemsa. These intracytoplasmic granulations stained with MayGrünwald-Giemsa could be "Gamma Bodies"' first described by Favre ${ }^{3}$ then Gamma. ${ }^{4}$ Mast cell and macrophage granulations were more easily distinguished by staining with May-Grünwald-Giemsa after immunoperoxidase. Similar granulations were also seen in the intercellular spaces (fig 2).

\section{DIRECT IMMUNOFLUORESCENT ANTIBODY STUDIES}

At low magnification $(\times 250)$, despite slight background fluorescence of the whole sections, fluorescent elements were seen clearly in the granulomatous areas. The number of fluorescent elements varied from one field to another in a given section and from one section to another in a given sample. These fluorescent elements occurred either alone or in clusters of two to 10. At higher magnification $(\times 400)$ the fluorescence was made up of smaller, irregularly shaped, clustered elements inside the cells. The cell nuclei were not fluorescent. In the granuloma fluorescent elements were also found outside the cells. Their specific fluorescence suggested their chlamydial nature (fig 3).

\section{IMMUNOPEROXIDASE STAINING}

In paraffin sections at low magnification $(\times 250)$ brown cells could easily be seen inside the granuloma. They predominated at the level of the nodular infiltrates. At higher magnification $(\times 400)$ the labelling was clearly inside the cytoplasm of macrophages. Pigment was found in clusters or was distributed outside the cells. The specific labelling of these elements suggested that they were chlamydial (fig 4). No labelling was noted in stained semithin sections.

\section{ELECTRON MICROSCOPY STUDIES}

In semithin sections (stained methylene blue azure II) the reticular dermal infiltrate was moderately dense and mainly composed of macrophages and lymphocytes. There were also some monocytes, neutrophil granulocytes, and plasma cells. Macrophages with large granulations were characteristic. Capillaries displayed a dilated cleft shape.

Only macrophages were seen in thin sections (counterstained with uranyle acetate and lead citrate) (fig 5). They were irregularly shaped and had many pseudopods. The Golgi apparatus was well developed. Mitochondria were numerous, and there were many lysosomes especially secondary lysosomes.

In most cells, different types of inclusions were found within the cytoplasm. Some were spherical $(0.3$ to $0.35 \mu \mathrm{m}$ in diameter), always included in a vacuole, and had one or several eccentric nucleoids.
These structures had ultrastructural features identical to those of elementary bodies (fig 6). Other inclusions were spherical $(0.5 \mu \mathrm{m}$ in diameter) with one or

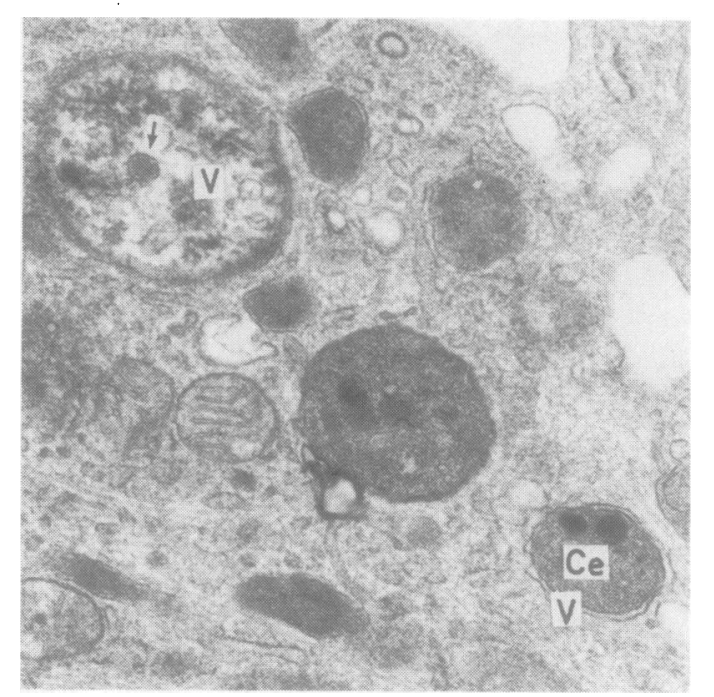

FIG 6 Elementary body with macrophage and osmiophil inclusion ( $\mathrm{Ce}=$ elementary body, $V=$ vacuole) $\rightarrow$ $(\times 3500)$.

several nucleoids in the centre of the structure. These structures were compatible with intermediate bodies. Further inclusions were very large (up to 2 or $3 \mu \mathrm{m}$ ) and were scattered throughout the cytoplasm. They were limited by a double unit membrane. They generally had a homogeneously dense electron content, although lamellar structures were occasionally seen. At the periphery of the cytoplasm a number of small nucleoids could be found. These inclusions were difficult to identify (fig 5 , insert). Initial bodies were rare. Only one binary fission was observed (fig 7). In two or three cells we noted one large vacuole limited by a single unit membrane containing some structures that had undergone necrosis.

\section{Discussion}

Our investigation concerned a case of LGV diagnosed two years after the onset of symptoms. Although the patient had received antibiotics before consulting us, in our opinion the diagnosis of LGV was not in doubt. Indeed our positive findings confirmed this diagnosis whereas the negative results did not refute it, and it was supported by the clinical and histological findings. Positive specific serology $(1 / 40)$ was atypical but this could be related to previous treatment. As is well recognised in the advanced stages of LGV, the condylomatous lesions hardly 


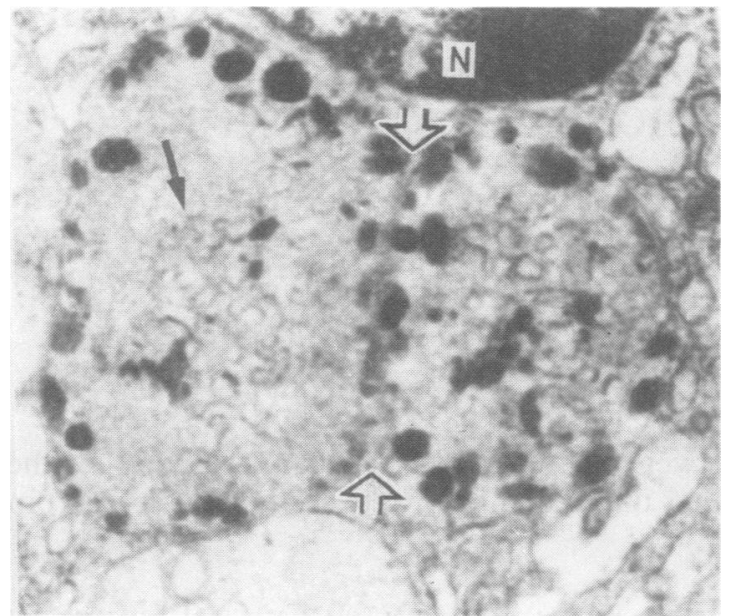

FIG 7 Atypical initial body in binary fission 9 .

Note filaments in central zone next to nucleus $\rightarrow$ $(\times 30000)$.

responded to treatment. Urethral culture for $C$ trachomatis gave negative results, but for technical reasons it could not be performed on granulomatous tissue. This negative result does not exclude the diagnosis, however, and other diagnoses are unlikely. In 1904 Favre $^{3}$ and $\mathrm{Gamma}^{4}$ found granulations within macrophages in histogical sections stained with May-Grünwald Giemsa and suggested that they could be all or part of the then unknown agent causing the disease. In the opinion of Sheldon and Heyman they were more likely to be the result of phagocytosis and not significant. ${ }^{5}$

In our study similar granulations were found on staining with May-Grünwald-Giemsa, immunofluorescence, immunoperoxidase, and on electron microscopy. Their topography inside the granuloma, intramacrophage site, size, shape, and staining were like those of the structures described in $1924 .^{34}$ The specificity of the immunological reactions confirmed that they were chlamydiae. Immunofluorescence specificity was checked by using two antibody and conjugate systems in parallel and by specific extinction studies. The failure of labelling by immunoperoxidase after removing Araldite from semithin sections can be explained by ethanolic sodium hydroxide having denatured antigenic sites.

On electron microscopy, however, there were not many intracytoplasmic inclusions displaying the classic ultrastructural features of chlamydiae that were described in culture by Eb et al. ${ }^{6}$ They do not account for the intense immunofluorescent and immunoperoxidase reactions. It may be that not only the classic chlamydial inclusions but also the atypical intracytoplasmic inclusions are both chlamydial bodies. Although the ultrastructure of the latter may be modified by the granulomatous reaction, antibiotic treatment, or both, these atypical inclusions may retain their antigenicity.

A study of the action of penicillin on Chlamydia psittaci showed that it has an effect on the binary fission of initial bodies, causes abnormal forms to appear, and modifies the ultrastructural aspect of the elementary body wall. ${ }^{7}$ Philipp and Metz studied the ultrastructural aspects of $C$ trachomatis isolated from a patient with LGV during the initial stage of the disease and before treatment and noted the normal cycle of development of chlamydiae with their characteristic structures. ${ }^{8}$ In our study tissue was removed for examination two years after the onset of clinical signs and after a number of antibiotic treatment regimens, which may explain the atypical features found.

As no similar study existed with which to compare our results on the immunocytology or ultrastructure of LGV, we wish merely to report our observations so that a number of hypotheses may be confirmed or refuted by further studies. Should these hypotheses be confirmed, a specific diagnosis of LGV could be made based mainly on the immunocytological study of biopsy specimens with well characterised antichlamydial antibodies; monoclonal antibodies would make this diagnosis more reliable and standardised. More accurate histological and ultrastructural knowledge of the cycle of chlamydiae in the granulomatous reaction would also be possible.

We suggest that direct immunofluorescence is a simple, rapid, and specific method for diagnosing LGV. Moreover, the advantage of this method is that it allows retrospective diagnosis on previous biopsies.

We thank Dr Y Gille of the bacteriology department, Hôpital de l'Antiquaille, Lyon and Dr Y Li of the histology department, Alexis Carrel Medical University, Lyon for their technical help.

\section{References}

1. Sainte Marie G. A paraffin embedding technique for studies employing immunofluorescence. J Histochem Cytochem 1962; 10:250.

2. Colimon R, Morel P. Maladie de Nicolas et Favre. In: Maladies infectieuses. Paris: Encyclopédie MedicoChirurgicale, 1979 (8076 A-10).

3. Favre M. Sur la lymphogranulomatose inguinale subaigue. $\mathbf{L a}$ Presse Médicale 1924;62:651-2.

4. Gamma C. Sur l'étiologie de la lymphogranulomatose inguinale subaigue. La Presse Médicale 1924; 37:404-5.

5. Sheldon WH, Heyman A. Lymphogranuloma venerum. A histologic study of the primary lesion, bubonulus, and lymph nodes in cases proved by isolation of the virus. Am J Pathol 1947; 23:653-65.

6. Eb F, Devauchelle G, Orfila J. Etude ultrastructurale de l'agent de la lymphogranulomatose vénérienne. Journal de Microscopie 1972; 13:47-56.

7. Matsumoto A, Mantre GP. Electron microscopic observation on the effects of penicillin on the morphology of Chlamydia psittaci. J Bactériol 1970; 101:278-85.

8. Philipp N, Metz J. Erregernachweiss bei lymphogranulomatosis inguinalis. Eine elecktron mikroskopishe undersuchung. Hautarzt 1975;26:411-5. 